\title{
An Efficient Way to Enhance Mammogram Image in Transformation Domain
}

\author{
T.A.Sangeetha, \\ M.Sc.,M.Phil., (PhD) \\ Assistant Professor in CS \\ Kongu Arts and Science college,Erode. \\ (Research Scholar-Mother Teresa Women's \\ University), \\ Kodaikkanal, TN,India.
}

\author{
A.Saradha., PhD. \\ Associate Professor \& H.O.D of CSE, \\ Institute of Road and Techology,Erode, TN, India.
}

\begin{abstract}
Breast cancer is one of the most important causes of increased women death rate in the world. Mammography is the most efficient approach for the early identification of breast diseases. The major objective of mammography is to identify small, non-palpable cancers during its premature stage. On the other hand, mammograms are extremely complicated to interpret being the fact that the pathological transformations of the breast are slight and their visibility is very poor with low contrast and noise. Mammograms have the valuable information such as microcalcifications and masses, which are extremely complicated to identify because mammograms are of low-contrast. Since the mammogram images are very noisy, low-contrast, blur and fuzzy, it is necessary to enhance the mammogram images for accurate identification and early diagnosis of breast cancer. In this paper, proposed an efficient technique to enhance the mammogram image using various transforms. The various transforms are wavelet transform, Curvelet transform, contourlet transform, Nonsubsampled transform. The drawback of wavelet transform is the method in which problem of filling missing data will occur. In Curvelet method the disadvantage is poor directional specificity of the images. In contourlet transform the image enhancement cannot capture the geometric information of images and tend to amplify noises when they are applied to noisy images since they cannot distinguish noises from weak edges. This entire drawback is overcome by the Nonsubsampled Contourlet transform. In order to determine the effectiveness of the proposed technique, experiments were carried using two UCI machine learning dataset and evaluated based on the PSNR value and MSE.
\end{abstract}

Keywords---Mammogram Images, Wavelet Transform, Curvelet Transform, Contourlet Transform, Non Subsampled Transform, and Image Enhancement.

\section{Introduction}

BREAST cancer is a "malignant neoplasm of the breast" [1]. The characteristic of cancer affected cells completely differs from the normal tissue cells in terms of the cell outline, shape, structure of nucleus and most significantly, its ability to metastasize and infiltrate. This disease is quite common. Because of its well exposed nature and possible for lethality, breast cancer is perhaps the most severe kind of cancer. Also, it is to be noted that, if identified and correctly treated during its early stages, breast cancer can be cured. Breast cancer continues to be a significant public health problem in the world, which not only endangers the life of the patient, but also causes damage to the female sexual characteristic organ
[1, 2, 3, and 4]. It's one of the most rapidly increasing malignant tumors. Early detection of breast cancer is of utmost importance: localized cancer leads to a 5-year survival rate of $97.5 \%$, whereas cancer that has spread to distant organs has a 5-year survival rate of only $20.4 \%$ [5]. Breast cancer can be either invasive or noninvasive [6].

\section{A. Noninvasive Breast Cancer}

Non-invasive cancers stay within the milk ducts or lobules in the breast. They do not grow into or overrun normal tissues within or beyond the breast. Non-invasive cancers are sometimes called carcinoma in situ ("in the same place") or pre-cancers shown in Figure 1.

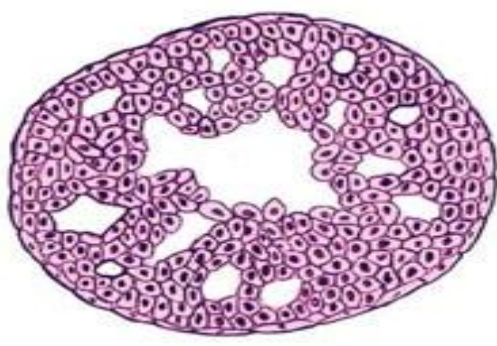

Fig 1: Non-Invasive Cell

\section{B. Invasive Breast Cancer}

Figure 2 shows the Invasive cancers grow into normal healthy tissues. Most breast cancers are invasive. Whether the cancer is non-invasive or invasive will determine your treatment choices and how you might respond to the treatments that you receive.

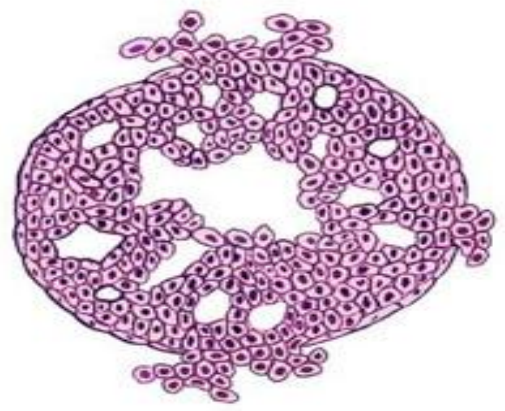

Fig 2: Invasive Cells 
In some cases, a breast cancer may be both invasive and non-invasive. This means that part of the cancer has grown into normal tissue and then a part of the cancer has stayed inside the milk ducts or milk lobules. It would be treated as an invasive cancer. A breast cancer also may be a "mixed tumor," that means it contains a fusion of cancerous ductal cells and lobular cells. This type of cancer is also called "invasive mammary breast cancer" or "infiltrating mammary carcinoma." It would be treated as a ductal carcinoma. If there is more than one tumor in the breast, the breast cancer is described as either multifocal or multicentric. In multifocal breast cancer, all of the tumors arise from the original tumor, and they are usually in the same section of the breast. If the cancer is multicentric, it means that all of the tumors formed separately, and they are often in different areas of the breast.

\section{Mammogram}

Mammograms are used as a screening tool to detect early stages of breast cancer in women experiencing no symptoms. It can also be used to detect and diagnose breast disease in women experiencing symptoms such as a lump, pain or nipple discharge. Mammography is the process of using low-energy$\mathrm{X}$-rays (usually around $30 \mathrm{kVp}$ ) to examine the human breast and is used as a diagnostic and a screening tool. The goal of mammography is the early detection of breast cancer, through detection of characteristic masses and/or microcalcifications. Like all $\mathrm{x}$-rays, mammograms use doses of ionizing radiation to create images. But the digital mammography is very noisy, low-contrast, blur and fuzzy and hence there is a requirement for enhancing Thus, there is a requirement to process an image on the image images [3]. This is essential for enhancing the Peak Signal-to-Noise Ratio (PSNR) and reducing the Mean Squared Error (MSE) for accurate identification. One of the most important objectives of mammogram image enhancement is to enhance the contrast between regions of interest and the background. Also the medical images fluctuate extensively in terms of acquisition, noise characteristics and quality [7] basis. This motivates the design and construction of effective mammogram image enhancement method using various transform. The remainder of this paper is organized as follows. The next section presents some basic concepts of mammography Section 3 provides a brief revision of various transform. Section 4 describes experimental results of various transform. Finally, the conclusions are drawn in Section 5.

\section{Related Work}

Mammography is one of the most reliable methods for early detection of breast carcinomas [6]. And it is currently the best way that can detect the clinical asymptomatic concealed breast cancer. It can reveal pronounced substantiation of abnormality, such as masses and calcifications, as well as slight signs such as bilateral asymmetry and architectural distortion [8]. Reports from the American Cancer Society (ACS) shows that early diagnosed breast cancer patient can achieve a survival rate as high as 97\% before the spreading of the carcinoma cells [9]. Therefore, early detection and diagnosis of the breast cancer is very important in saving the life of the patient and it's the most effective way to increase the cure rate and lower the mortality rate. However, because of the particularity of the breast tissue, mammograms are always lack of hierarchy, lowcontrast, leaving the misdiagnosis rate and neglected diagnosis rate too high. Since diagnostic features in mammograms, such as masses and calcifications, may be small and have low contrast with respect to the surrounding breast tissues. These attributes could render the diagnostic features hard to detect. Contrast enhancement techniques can improve the ability of a radiologist to perceive subtle diagnostic features, leading to earlier, more accurate diagnosis of breast cancer. Contrast enhancement can improve the quality of an otherwise unsatisfactory mammogram, as stated by Ram [10], who further indicated that the application of contrast enhancement techniques in a clinical situation may reduce the radiation dose by about $50 \%$. The enhancement of mammographic images could improve the accuracy of detection of early signs of breast features. To highlight the features of the lesion region and improve the visual effect of the mammogram, the most commonly used method is imposing enhancement preprocessing on the image [11, 12]. For reviews on image enhancement in mammography, see Rangayyan [13], Morrow et al. [14], and Rangayyan et al. [15]. Currently, contrast stretching, histogram equalization spatial domain filtering, frequency domain filtering, Wavelet Transform (WT), mathematical morphology etc. are the major commonly used image preprocessing techniques [16, 17-22, 23, 24]. Although those techniques have achieved good enhancement results to some extent, however, it is still far from being satisfactory. Multiscale Geometric Analysis (MGA) is one of the research focuses on image enhancement $[25,26]$. MGA not only possesses the main features of wavelets, namely, multiscale and time-frequency localization, but also offers a high degree of directionality and anisotropy, so it gets widely used and developed. Up to now, many MGA methods have been proposed, such as Ridgelet [27], Curvelet [28], Bandelet [29], Contourlet [30] etc. They have been applied in a several image processing task and achieved good effectiveness. In this paper, a mammographic image enhancement method based on shift invariant .In this paper, present the nonsubsampled contourlet transform (NSCT), which is a shift-invariant version of the contourlet transform. The NSCT is built upon iterated nonsubsampled filter banks to obtain a shift-invariant directional multiresolution image representation. Based on the NSCT, propose a new method for image enhancement.

\section{A Revision of Various Transform}

\section{A. Wavelet Transform}

The wavelet transform (WT) is a powerful mathematical tool, with several applications in computer graphics and image processing. The wavelet analysis is done by applying a function called the mother wavelet (usually denoted by $\psi(\mathrm{x})$ ) to a signal/image, which allows one not only to identify its frequency components, but also the spatial location where these components appear. The notion of scale is very important in the wavelet theory: the coarser the scale of analysis, the fewer details of the signal is caught. On the other hand, finer scales capture more details of the processed signal/image. This section presents our model for digital enhancement in the wavelet domain. It explores wavelet coefficients to ensure the proper preservation of the image structure and the correct filling of the enhanced region with block-based texture synthesis. The method takes an image I and a user defined inpainting mask $\Omega$ as input, and decomposes both images using a decimated WT. Wavelet 
Coefficient are then propagated into the enhance region, and the inverse WT is applied to obtain the final reconstruction image. For wavelet coefficient propagation into the enhance region, there are two important steps that must be executed at each iteration in the algorithm. After the data have been prepared (this step will be explained later), the proposed model iteratively fills the inpainting region, until it is completely filled. At each iteration, a block with varying size is selected as the filling target, based on its geometric aspects and the energy of the wavelet coefficients in neighboring regions. Once this block is determined, the patch that will fill it must be selected considering the structural aspects and the texture in the neighborhood of the inpainting block. The next sections detail the inner steps of this model, especially the proposed approach for determining the priority of inpainting blocks and the metric used in the block-based wavelet texture synthesis.

The wavelet Transform in which, it process the digital enhancement of mammogram images in the wavelet domain. In the wavelet transform in which it consist of many steps like data presentation, searching of the best block to fill, Edge strength, edge orientation, the confidence term etc. The drawback of wavelet transform is the method in which problem of filling missing data will occur and the PSNR value is very low. So we go for Curvelet transform.

\section{B. Curvelet Transform}

The Curvelet Transform is based, on decomposing the image into different scales, then partitionining into squares whose sizes based on the corresponding scale. The curvelet decomposition can be stated in the following form [31].

First, Subband Decomposition. $f$ is filtered into subbands,

$$
f \rightarrow\left(P_{0}, \Delta f_{1}, \Delta f_{2}, \Delta f_{a}\right)
$$

Second, Smooth Partitioning. Each subband is smoothly windowed into "squares" of an appropriate scale $\Delta_{s} f \rightarrow w_{Q} \Delta_{s} f$

The Curvelet transform have a new kind pyramid structure. First the pyramid, indexed by $\mathrm{Q}$ whose range is recalled to be the set of all dyadic squares, which localizes the image both in space and frequency.

Second pyramid, the Ridgelet pyramid which analyzes each renormalized block of image data that obey spatial and frequency localization.

The Curvelet transform is suited for images contains edges, so it is a good for edge enhancement. Curvelet coefficients can by modified in order to enhance edges in an image by $\mathrm{y}(\mathrm{x})$ (Velde function). This function aims to faint edges, the formula is defined by:

$$
y(x)=\left[\frac{m^{p}}{c}\right] \text { if }|x|<c
$$

Three parameters used : p, m, and c. p determines the degree of non-linearity and must be in $[0,1]$. Coefficients larger than $\mathrm{m}$ are not modified by algorithm. The c parameter corresponds to the noise level.

Curvelet transform has new features like the scaling law, in other words, the spatial domain related with scale by parabolic curving. Also it has new pyramid. The enhancement on curvelet transform can obtain good results, especially for images having curve edges. But the directional specificity of the image is poor due to that the efficiency is poor.

\section{Contourlet Transform}

The contourlet transform is an extension of wavelet transform in two dimensions, which has been introduced by Minh Do and Martin Vetterli (Duncan, et al., 2006) the contourlet transform combines Laplacian Pyramid (LP) with a Directional Filter Bank (DFB). The Laplacian pyramid is first used to capture the point discontinuities, and is then followed by a directional filter bank to link point discontinuities into linear structures. The Laplacian Pyramid (LP) is used to decompose an image into a number of radial subbands and the Directional Filter Banks (DFB) decompose each LP detail subband into any power of two's number of directional subbands. Fig. 1shows an example frequency partition of the contourlet transform where the three scales are divided into four, eight and eight directional subbands from coarse to fine scale, respectively. The contourlet coefficient can be represented in a quad-tree structure. Each coefficient in the coarsest level has four children in the next higher subband and each of the children has four children in the next higher subband and a quad -tree will emerge.

In Contourlet transform in which, the image is said to be a contourlet it sets the coefficient values then it go for image enhancement process.

\section{a) Contourlet Based Enhancement}

The contourlet based enhancement is used to reconstruct the enhanced image from the modified contourlet coefficient is as follows

\section{i. Contourlet Transform and Counter Coefficient}

Fig 3(a) shows the flow graph of contourlet transform. It consists of two steps: the subbands decomposition and the directional transform. A Laplacian pyramid (LP) is first used to capture point discontinuities, then followed by a directional filter bank (DFB) to link point discontinuity into linear structure. The overall result is an image expansion using basic elements like contour segments, and is thus named the Contourlet.

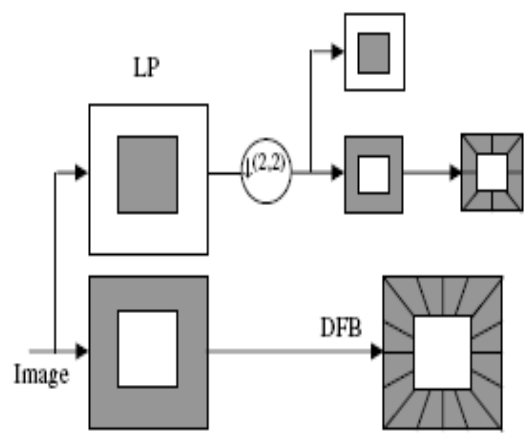

\section{Fig 3(a): A Flow Graph of Contourlet Transforms}

In Fig 3(a) it shows a flow graph of contourlet transform. The Image is first decomposed into subbands through Laplacian Pyramid and then each band pass detail image is analyzed by the directional filter banks.

\section{ii. Algorithm with Contour Based Enhancement}

Contourlet transform is well-adapted to represent images containing edges it is a good candidate for microstructure enhancement in images as well as edge enhancement in natural images. Contourlet coefficients can be modified via a 
nonlinear function $\mathrm{y}_{\alpha}$. Taking noise into consideration, we introduce explicitly a noise standard deviation $\sigma$ in the equation

$$
\begin{aligned}
& y_{1} \alpha(x, \sigma)=1 \text { if } x<\alpha( \\
& y_{\alpha}(x, \sigma)=\frac{x-\omega \sigma}{\alpha \sigma} \cdot\left(\frac{t}{\alpha \sigma}\right)^{q}+\frac{2 \alpha \sigma-x}{\alpha-\sigma} \text { if } \sigma \leq \mathrm{x}<2 \alpha \sigma \\
& y_{1}(\alpha(x, \alpha)=(t / x)) q \quad \text { if } 2(\alpha \leq \mathrm{x}<t \\
& y_{\alpha(\alpha, \sigma)}=\left(\frac{t}{x}\right)^{s} \quad \text { if } \mathrm{x} \geq \mathrm{t}
\end{aligned}
$$

Here, $t$ determines the degree of nonlinearity and $s$ introduces a dynamic range compression. Using a nonzero $\mathrm{s}$ will enhance the faintest edges and soften the strongest edges. $\alpha$ is a normalization parameter. The $t$ parameter is the value under which coefficients are amplified. This value depends obviously on the pixel values. We can derive the $t$ value from the data. Two options are possible:

1) $t=F_{t} \sigma$, where $\sigma$ is standard noise deviation and $F_{t}$ is an additional parameter which is independent of the Contourlet coefficient values, and therefore much easier for a user to set. For instance, using $\alpha=3$ and $F_{t}=10$ amplifies all coefficients between 3 and 30 .

2) $\mathrm{t}=1 \mathrm{M} \alpha$, with $1<1$, where $\mathrm{M} \alpha$ is the maximum Contourlet coefficient of the relative band. In this case, choosing for instance $\alpha=3$ and $1=0.5$, we amplify all coefficients with an absolute value between $3 \sigma$ and half the maximum absolute value of the band.

The first choice allows the user to describe the coefficients to be amplified as a function of their signal to noise ratio, while the second one gives an easy and general way to fix $t$ independently of the range of the pixel values.

The Contourlet transform has better performance in representing edges than wavelets, and the Curvelet transform, therefore well-suited for multi-scale edge enhancement. The transform which gives a good visual quality of the images but for image enhancement cannot capture the geometric information of images and tend to amplify noises when they are applied to noisy images since they cannot distinguish noises from weak edges. In contrast, the nonsubsampled contourlet transform extracts the geometric information of images, which can be used to distinguish noises from weak edges so we go for non subsampled contourlet transform.

\section{Non Subsampled Contourlet Transform}

Due to downsamplers and upsamplers present in both the LP and the DFB, the contourlet transform is not shiftinvariant, which will cause pseudo-Gibbs phenomena [32] around singularities. In 2006, L. Da Cunha and J. Zhou [33] improved the Contourlet Transform and developed the nonsubsampled contourlet transform. Figure 4 displays an overview of the proposed NSCT. The NSCT is a fully shift-invariant, multiscale, and multidirectional expansion that has a fast implementation. It is composed of two shift-invariant parts: 1) a nonsubsampled pyramid structure that ensures the multiscale property and 2) a nonsubsampled DFB structure that gives directionality, without sampling as that in the Contourlet Transform. Because of its shiftinvariant property, can achieve better results in the image processing tasks where redundancy is not a major issue, such as image denoising and enhancement. Besides, it is more flexible for the design of the filter.

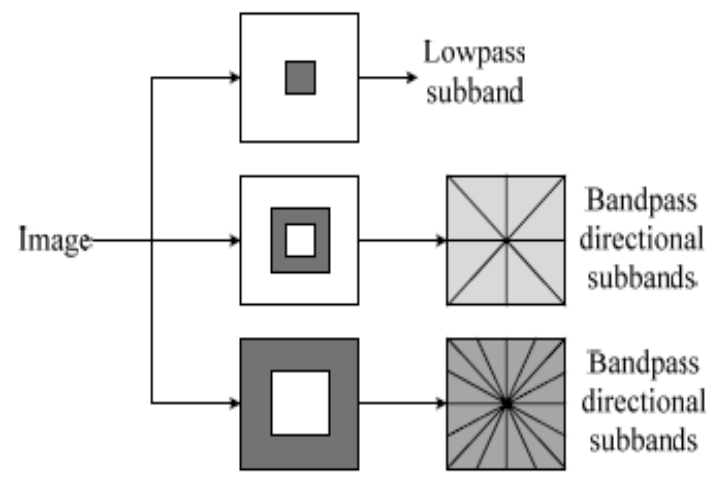

Fig 4: The Flowchart of NSTC

\section{E. Steps to Enhance the Mammogram Image using Non Subsampled Transform}

The mammogram images are very noisy, low-contrast, blur and fuzzy, hence there is a requirement for image enhancement.

Initially, the mammogram images are transformed to preprocessing process for preliminary enhancement. Then the output of preprocessing is given as input to the nonsubsampled contourlet transform.

In this transform, the preprocessing is not used as the preliminary step, even though it is more efficient. Because it enhances the image contrast by transforming the pixel distribution as a result they can conform to uniform distribution.

The next step is image enhancement in which the algorithm is using for enhance the images. First, the strong edges correspond to those pixels with big-value coefficients in all subbands. Second, the weak edges correspond to those pixels with big-value coefficients in some directional subbands but small-value coefficients in other directional subbands within the same scale. Finally, the noises correspond to those pixels with small-value coefficients in all subbands. Based on this observation, classify pixels into three categories by analyzing the distribution of their coefficients in different subbands. One simple way is to compute the mean (denoted by mean) and the maximum (denoted by max) magnitude of the coefficients for each pixel, and then classify it by

$$
\left\{\begin{array}{l}
\text { strong edge if mean } \geq c \sigma \\
\text { weak edge if mean }<c \sigma_{w} m a x \geq c \sigma_{v} \\
\text { noise if mean }<c \sigma_{s} \text { maxi }<c \sigma
\end{array}\right.
$$

Where $\mathrm{c}$ is a parameter ranging from 1 to 5 , and is the noise standard deviation of the subbands at a specific level.

The NSCT is shift-invariant so that each pixel of the transform subbands corresponds to that of the original image in the same spatial location. Therefore, gather the geometrical information pixel by pixel from the NSCT coefficients.

The mixture model differentiates the coefficients into three categories without using explicit threshold values. By specifying amplification factors for each category, derive a non-linear mapping function in this section. From this mapping function it is evident that the mid-range coefficients are amplified the most. In nonsubsampled contourlet transform a nonsubsampled pyramid split the input into a lowpass subband and a highpass subband.

Then a nonsubsampled DFB decomposes the highpass subband into several directional subbands. The scheme is iterated repeatedly on the lowpass subband a reconstruction image of NSTC will formed. Finally the result obtained is 
enhanced images with clarity and free from noise. The transform in which it suppresses noises while enhancing weak edges in the textures and boosting the contrast between the lesion area and the background.

\section{Experimental Results}

For the evaluation of the proposed mammogram image enhancement technique using various transform, two datasets are taken from the UCI Machine Learning Repository. The datasets are

- Wisconsin Diagnostic Breast Cancer (WDBC)[34] and

- $\quad$ Breast Cancer Dataset [35]

Besides, the quality of the images are evaluated using the traditional distortion measurements such as

- Mean Squared Error (MSE) and

- Peak Signal-to-Noise Ratio (PSNR)

Mean Squared Error

MSE of the output image is defined as

MSE $=\frac{\sum_{i=1}^{M} \sum_{j=1}^{N}|x(i, j)-\hat{\lambda}(i, j)|^{2}}{M N}$

where $x(i, j)$ is the original image, $x(i, j)$ is the output image, and $M N$ is the size of the image.

MSE is calculated for all the three approaches. Figure 4.1 shows the comparison of the MSE for the proposed method with the adaptive histogram equalization and wavelet domain.

PSNR

PSNR is defined as

$P S N R=20 \log _{10}\left[\frac{\left(2^{\mathrm{m}}-1\right)}{\sqrt{M S E}}\right](d B)$

where $n$ is the number of bits used in representing the pixel of the image.

PSNR is calculated for all the approaches.

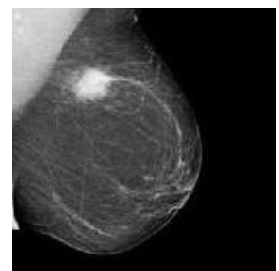

(a) Original Image

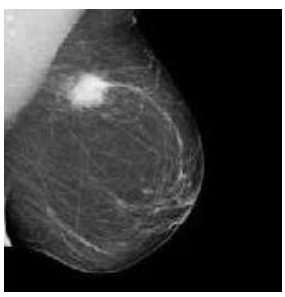

(a) Original Image

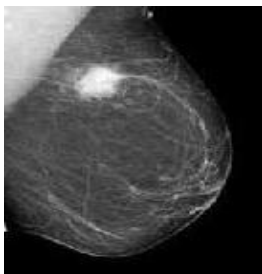

(b) Enhanced Image using Wavelet Transform

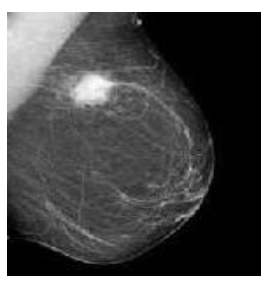

(b) Enhanced Image using Curvelet Transform

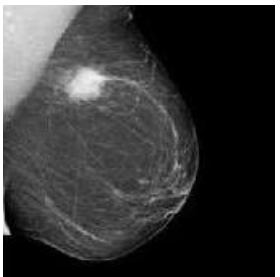

(a) Original Image

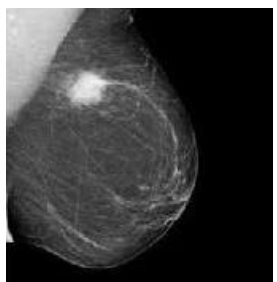

(a) Original Image

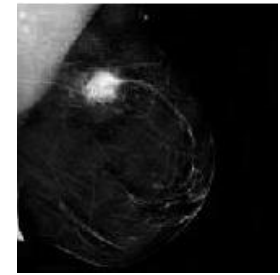

(b) Enhanced Image using Contourlet Transform

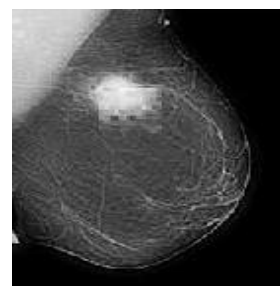

(b) Enhanced Image using Non Subsampled Contourlet Transform

\section{Fig 5: Resultant Image of Various Transform}

From the Figure 5, observe that all the transform in which the nonsubsampled contourlet Transform is the best and more efficient method to enhance the images. It suppresses noises while enhancing weak edges in the textures and boosting the contrast between the lesion area and the background.

Table 1: Comparison of PSNR and MSE Values for

\begin{tabular}{|l|c|c|}
\multicolumn{3}{c}{ Various Transform } \\
$\begin{array}{l}\text { Various } \\
\text { Transform }\end{array}$ & $\begin{array}{c}\text { PSNR } \\
\text { Values(dB) }\end{array}$ & MSE Values \\
\hline Wavelet Method & 17.239 & 70.89 \\
\hline Curvelet Method & 27.239 & 53.28 \\
\hline $\begin{array}{l}\text { Contourlet } \\
\text { Method }\end{array}$ & 39.202 & 33.52 \\
\hline $\begin{array}{l}\text { Nonsubsampled } \\
\text { Contourlet } \\
\text { Method }\end{array}$ & 45.039 & 11.01 \\
\hline
\end{tabular}

From the table 1, shows the values of PSNR and MSE values of the various transforms. From that the nonsubsampled transform is reliable and accurate one for image enhancement.

\section{Conclusion}

At present, one of the most important causes of cancer death among middle aged women is breast cancer. Mammography is technique used by radiologists for early detection and diagnosis of cancer in breast images. Digital mammogram has turned out to be the most effective technique for premature breast cancer detection. Digital mammogram captures an electronic image of the breast and accumulates it in a computer. Mammogram images are very noisy, lowcontrast, blur and fuzzy and hence the mammogram images are enhanced for accurate identification of breast cancer. Processing these images require high computational capabilities. In this paper, the mammogram images are enhanced using various transform. It is found from the experiments that the image enhancement method using nonsubsampled contourlet transform is efficient while 
comparing with other methods and useful in capturing relevant clinical information since its PSNR value is high with very low MSE value.

\section{REFERENCES}

[1] Temidayo O Ogundiran, Samuel A Ademola, Odunayo M Oluwatosin, Effiong E Akang and Clement A Adebamowo, "Primary osteogenic sarcoma of the breast”, World Journal of Surgical Oncology, Published online: http://www.biomedcentral.com/content/pdf/14777819-4 90.pdf, 2006.

[2] Breast Cancer In-Depth Report, The Newyork Times, http://health.nytimes.com/health/guides/disease/breast cancer/print.html.

[3] Keir Bovis and Sameer Singh, "Enhancement Technique Evaluation using Quantitative Measures on Digital Mammograms", Proc. 5th International Workshop on Digital Mammography, Toronto, Canada, M.J. Yaffe (ed.), Medical Physics Publishing, Pp. 547-553, 2000.

[4] R. Krishnamoorthy, N. Amudhavalli and M.K. Sivakkolunthu, "An Adaptive Mammographic Image Enhancement in Orthogonal Polynomials Domain", International Journal of Computer and Information Engineering, Vol. 4, No. 2, Pp. 120-128, 2010.

[5] C. D. Maggio, "State of the art of current modalities for the diagnosis of breast lesions," European Journal of Nuclear Medicine and Molecular Imaging, vol. 31, pp. $56-69,2004$

[6] Breast Cancer In-Depth Report, The Newyork Times, http://health.nytimes.com/health/guides/disease/breast cancer/print.html.

[7] R. Krishnamoorthy, N. Amudhavalli and M.K. Sivakkolunthu, "An Adaptive Mammographic Image Enhancement in Orthogonal Polynomials Domain", International Journal of Computer and Information Engineering, Vol. 4, No. 2, Pp. 120-128, 2010.

[8] M. J. Homer, Mammographic Interpretation: A Practical Approach. New York: McGraw-Hill Companies, 1991.

[9] ACS, "Cancer Prevention \& Early Detection Facts \& Figures 2008," 2008.

[10] G. Ram, "Optimization of ionizing radiation usage in medical imaging by means of image enhancement techniques," Medical Physics, vol. 9, pp. 733-737, 1982.

[11]H. D. Cheng, X. Cai, X. Chen, L. Hu, and X. Lou, "Computer-aided detection and classification of microcalcifications in mammograms: a survey," Pattern Recognition, vol. 36, pp. 2967-2991, 2003.

[12]H. D. Cheng, X. J. Shi, R. Min, L. M. Hu, X. P. Cai, and H.N. Du, "Approaches for automated detection and classification of masses in mammograms," Pattern Recognition, vol. 39, pp. 646-668, 2006.

[13]R. M. Rangayyan, Biomedical Image Analysis. Boca Raton, FL: CRC Press, 2005.

[14] W. M. Morrow, R. B. Paranjape, R. M. Rangayyan, and J.E. L. Desautels, "Region-based contrast enhancement of mammograms," IEEE Trans. Med. Image., vol. 11, pp. 392-406, 1992.
[15] R. M. Rangayyan, L. Shen, Y. Shen, J. E. L. Desautels, H.Bryant, T. J. Terry, N. Horeczko, and M. S. Rose, "Improvement of sensitivity of breast cancer diagnosis with adaptive neighborhood contrast enhancement of mammograms," Information Technology in Biomedicine, IEEE Transactions on, vol. 1, pp. 161-170, 1997.

[16]D. C. Chang and W. R. Wu, "Image contrast enhancement based on a histogram transformation of local standard deviation," IEEE Trans. Med. Imag., vol. 17 , pp. $518531,1998$.

[17]H. D. Cheng, X. Cai, X. Chen, L. Hu, and X. Lou, "Computer-aided detection and classification of microcalcifications in mammograms: a survey," Pattern Recognition, vol. 36, pp. 2967-2991, 2003.

[18]H. D. Cheng, X. J. Shi, R. Min, L. M. Hu, X. P. Cai, and H.N. Du, "Approaches for automated detection and classification of masses in mammograms," Pattern Recognition, vol. 39, pp. 646-668, 2006.

[19] H. K. Kang, Y. M. Ro, and S. M. Kim, "A Microcalcification Detection Using Adaptive Contrast Enhancement on Wavelet Transform and Neural Network," IEICE Transactions on Information and Systems, pp. 1280-1287, 2006.

[20]H.-K. Kang, N. N. Thanh, S.-M. Kim, and Y. M. Ro, "Robust Contrast Enhancement for Microcalcification in Mammography," in Computational Science and Its Applications - ICCSA 2004, 2004, pp. 602-610.

[21]H.-K. Kang, S.-M. Kim, N. N. Thanh, Y. M. Ro, and W.H. Kim, "Adaptive Microcalcification Detection in Computer Aided Diagnosis," in Computational Science ICCS 2004, 2004, pp. 1110-1117.

[22] H. Li, K. J. Liu, S. C. B. Lo, O. T. Inc, and M. D. Jessup, "Fractal modeling and segmentation for the enhancement of microcalcifications in digital mammograms," IEEE Trans. Med. Imag., vol. 16, pp. 785-798, 1997.

[23] P. Sakellaropoulos, L. Costaridou, and G. Panayiotakis, "A wavelet-based spatially adaptive method for mammographic contrast enhancement," Physics in Medicine and Biology, vol. 48, pp. 787-803, 2003.

[24] V. E. Pera, E. L. Heffer, H. Siebold, O. Schütz, S. Heywang-K? brunner, L. G?tz, A. Heinig, and S. Fantini, "Spatial second-derivative image processing: an application to optical mammography to enhance the detection of breast tumors," Journal of Biomedical Optics, vol. 8, p. 517,2003.

[25] J. K. Romberg, M. B. Wakin, and R. G. Baraniuk, "Multiscale geometric image processing," in Proceedings of the SPIE: Visual Communications and Image Processing 2003, 2003, pp. 1265-1272.

[26] J. Li-Cheng and T. Shan, "Development and prospect of image multiscale geometric analysis," Acta Electronica Sinica, vol. 31, pp. 1975-1981, 2003.

[27]M. N. Do and M. Vetterli, "The finite Ridgelet transform for image representation," IEEE Trans. Image Process, vol.12, pp. 16-28, 2003.

[28] J. L. Starck, E. J. Candes, and D. L. Donoho, "The Curvelet transform for image denoising," IEEE Trans. Image Process, vol. 11, pp. 670-684, 2002. 
[29] Giovanni Luca Masala, "Computer Aided Detection on Mammography", World Academy of Science, Engineering and Technology, Vol. 15, Pp. 1-6, 2006.

[30] M. N. Do and M. Vetterli, "The contourlet transform: an efficient directional multiresolution image representation," IEEE Trans. Image Process, vol. 14, pp. 2091-2106, 2005.

[31] Starck , Murtagh ,E.J Candes ,D.L. Donoho, "Gray and Color Image Contrast Enhancement by the Curvelet Transform," IEEE Transactions on Image Processing .vol., 12, pp. 706- 716, June 2003.
[32] R. R. Coifman and D. L. Donoho, Translation invariant de-noising: Wavelets and statistics. Newyork: Springer Verlag, 1995.

[33] A. L. Da Cunha, J. Zhou, and M. N. Do, "The Nonsubsampled Contourlet Transform: Theory, Design, and Applications," IEEE Trans. Image Process, vol. 15, pp. 3089-3101, 2006

[34]Zimmerman, J.B., Pizer, S.M., "An evaluation of the effectiveness of adaptive histogram equalization for contrast enhancement”, IEEE Trans Med. Imaging, Vol. 7, No. 4, Pp. 304-312, 1988.

[35] http://archive.ics.uci.edu/ml/datasets/Breast+Cancer+Wis consin+\%28Diagnostic\%29 